\title{
Multiple description coding with side information for stereoscopic 3D
}

\begin{abstract}
Stereoscopic video as the simplest form of 3D video is already being used in consumer devices such as 3DTV and 3D mobile phone. When the 3D video from the 3D mobile phone is compressed and transmitted over error prone channels, error propagation due to packet loss leads to poor 3D visual quality. The objective of the paper is to provide error resilience 3D video using the well known multiple description coding (MDC) technique. Specifically, the MDC is modified for 2D plus depth stereoscopic video with the addition of spatially reduced resolution of the side information. The proposed method reduces the depth bit rates and consequently: 1) improves their rate distortion, particularly at higher bit rates in error free channels; and 2) improves their performance in ideal MDC channel.
\end{abstract}

Keyword: Stereoscopic 3D video; Multiple description video coding; Side information; Reduced resolution; Error resilience 